\title{
Semi-Automated Unidirectional Sequence Analysis for Mutation Detection in a Clinical Diagnostic Setting
}

\author{
Sian Ellard, ${ }^{1,2}$ Beverley Shields, ${ }^{2}$ Carolyn Tysoe, ${ }^{1}$ Rebecca Treacy, ${ }^{3}$ \\ Shu Yau, ${ }^{4}$ Christopher Mattocks, ${ }^{5}$ and Andrew Wallace ${ }^{6}$
}

Background: The past 10 years have seen an improvement in sequence data quality due to the introduction of capillary sequencers and new sequencing chemistries. In parallel, new software programs for automated mutation detection have been developed. We evaluated the sensitivity of semiautomated unidirectional sequence analysis for the detection of heterozygous base substitutions using the Mutation Surveyor software package. Methods: Detection rates for heterozygous base substitutions in 29 genes by automated and visual inspection were compared. Examples of heterozygous bases not detected in one direction during bidirectional analysis were also sought through a national survey of United Kingdom (UK) genetics laboratories. Sequence quality was assessed in a consecutive cohort of 50 patients for whom the 39 exons of the $A B C C 8$ gene had been sequenced in one direction. Results: A total of 701 different heterozygous base substitutions were detected by the software with no false negatives (sensitivity $\geq 99.57 \%$ ). Four examples of heterozygous bases missed in one direction during bidirectional analysis were reported. Two were detected using unidirectional analysis settings, and the other two bases had low-quality scores. Of the 1950 amplicons examined, $97.2 \%$ had a quality score $\geq 30$ and an average PHRED-like score $\geq 50$ for the defined region of interest, and $98.1 \%$ of the 323,650 bases had a PHRED score $>40$. Conclusions: We found no evidence to support a requirement for bidirectional sequencing. Semiautomated analysis of good quality unidirectional sequence data has high sensitivity and is suitable for heterozygote mutation scanning in clinical diagnostic laboratories. Further work is required to determine minimum quality parameters for semiautomated analysis.

\section{Introduction}

M OLECULAR GENETIC TESTING IS WIDELY USED to confirm the clinical diagnosis of a genetic disorder, to determine carrier status and for presymptomatic or prenatal testing. The completion of the human genome sequence in 2003 facilitated the identification of gene mutations causative of human disease, and mutations in $>2000$ genes have now been identified in patients with more than 3000 different disease phenotypes (McKusick, 2007). Genetic testing is available for more than 300 single-gene disorders through the United Kingdom (UK) Genetic Testing Network (www.ukgtn.nhs.uk), and the majority of tests require mutation scanning of one or more genes.

There are many different techniques for mutation scanning, but essentially there are two approaches: a prescreen that detects but does not characterize variants, and direct sequence analysis. Prescreen approaches historically include singlestrand conformational polymorphism, chemical cleavage of mismatch, and various forms of heteroduplex analysis of which denaturing high performance liquid chromatography, conformation-sensitive capillary electrophoresis, and highresolution melt-curve analysis are most frequently employed in the diagnostic laboratory (Steensma, 2006). However, sequencing is widely used for mutation scanning and allows the detection and characterization of mutations in a single step.

Sequencing analysis for mutation scanning has traditionally been performed by sequencing double-stranded PCR products in both the forward and reverse directions. However, there is no known biological reason why a mutation would be visible in only one direction, and there are examples where bidirectional sequence analysis is not achievable due to polymorphic regions. For example, individuals heterozygous

\footnotetext{
${ }^{1}$ Department of Molecular Genetics, Royal Devon \& Exeter NHS Foundation Trust, Exeter, United Kingdom.

${ }^{2}$ Institute of Biomedical and Clinical Science, Peninsula Medical School, Exeter, United Kingdom.

${ }^{3}$ East Anglian Medical Genetics Service, Addenbrooke's Hospital, Cambridge, United Kingdom.

${ }^{4}$ DNA Laboratory, Guy's \& St. Thomas' NHS Foundation Trust, London, United Kingdom.

${ }^{5}$ National Genetics Reference Laboratory (Wessex), Salisbury District Hospital, Salisbury, United Kingdom.

${ }^{6}$ National Genetics Reference Laboratory (Manchester), St Mary's Hospital, Manchester, United Kingdom.

On behalf of the Scientific Sub-Committee of the Clinical Molecular Genetics Society.
} 
for the CFTR intron $8 \mathrm{TG}_{\mathrm{n}}$ or polyT tract polymorphisms will show frameshifted exon 9 sequence in the forward direction.

When sequencing was first adopted for mutation screening in the mid-1990s, analysis in both directions was necessary to achieve maximum sensitivity. At this time heterozygote detection relied on detection of a secondary peak, and sequencing chemistries produced traces with a significant context-dependent peak height variation. The combination of these factors meant that in some cases a secondary peak was only high enough to be detected in one of the sequencing orientations. Sequence quality and uniformity has improved with the introduction of capillary sequencers and new chemistries, and many software analysis packages now incorporate quality parameters such as PHRED scores to provide a measure of sequence data quality. Two-directional sequencing is often employed to achieve complete coverage of the region of interest (ROI) (the exon plus conserved splice sites), but although most clinical diagnostic laboratories perform bidirectional sequencing, the analysis may be unidirectional in part.

A number of commercial software packages (e.g., Mutation Surveyor, SeqScape, Sequence Pilot, and Sequencher) have been developed for automated detection of heterozygous and homozygous mutations. In this study we evaluated the Mutation Surveyor package whose manufacturer (SoftGenetics, Philadelphia, PA) claims a sensitivity of $\geq 99 \%$ for bidirectional and $\geq 95 \%$ for unidirectional sequencing. A previous evaluation of Mutation Surveyor v2.51 for bidirectional analysis of heterozygous or homozygous mutations reported a false-negative rate of $0-1.1 \%$ (Patel and Wallace, 2005), but additional false-negative results were observed for poor quality data. Evidence from the European Molecular Genetics Quality Network (www.emqn.org) sequencing quality assurance schemes suggests that data quality is highly variable between laboratories. In the 2003 scheme, $64 \%$ of sequence data from 57 laboratories exceeded a PHRED score of 40, but for individual centers this figure ranged from $15 \%$ to $84 \%$ (Patton et al., 2006).

In this study we evaluated the sensitivity of unidirectional sequence analysis using Mutation Surveyor compared to visual inspection for the detection of heterozygous base substitutions. We also sought examples of heterozygous base substitutions not detected by the software in a bidirectional analysis through a national survey of UK laboratories (coordinated by the Clinical Molecular Genetics Society). Finally, we used Mutation Surveyor v3.10 to assess data quality and the detection of variants within the 39 exons of the $A B C C 8$ gene in a consecutive series of 50 patients with neonatal hyperinsulinism or diabetes. Loss-of-function mutations in this gene are associated with congenital hyperinsulinism (Thomas et al., 1995, 1996), and activating mutations cause permanent or transient neonatal diabetes (Ellard et al., 2007; Flanagan et al., 2007).

\section{Materials and Methods}

\section{Unidirectional sequence analysis for heterozygous base substitutions}

Unidirectional sequence data generated from clinical referrals during a 2-year period was collated for a total of 29 genes tested in three laboratories (Laboratory 1, VHL, APC, MLH1, MSH2, SLC12A3, UMOD, and MYH9; Laboratory 2, SPG4, SPG3A, BRCA1, BRCA2, DMD, and RYR1; Laboratory 3, GCK, HNF1A, HNF4A, HNF1B, NOTCH3, KCNJ11, ABCC8,
MEN1, RET, LMNA, JAG1, EIF2AK3, PHEX, GLUD1, INS, and CFTR). Sequences were analyzed using Mutation Surveyor v3.10 with the "Basepatch" option selected, "Allow Computer to Delete Mutations" unchecked, and all other functions, including sensitivity (high), at their default settings. The number of unique heterozygous base substitutions detected was recorded. These substitutions were classified as pathogenic mutations (reported to the referring clinician as consistent with or confirming the clinical diagnosis), single-nucleotide polymorphisms (SNPs), or variants of uncertain clinical significance (unclassified). A visual inspection of the electropherograms and difference traces was also performed to identify any false-negative results. The data set was restricted to heterozygous mutations; homozygous, hemizygous, and mosaic mutations were not recorded. Frameshift mutations were recorded but not included in the calculation to estimate the sensitivity of the software for the detection of heterozygous base substitutions.

\section{Bidirectional sequence analysis for heterozygous base substitutions}

Examples of missed mutations or polymorphisms were identified through a national survey of UK laboratories using a bidirectional sequencing approach.

\section{Analysis of unidirectional ABCC8 sequence data}

A consecutive series of 50 patients with either congenital hyperinsulinism or neonatal diabetes were investigated. The ABCC8 gene (sequence accession numbers U63421 and L78208) has 39 exons and encodes the SUR1 protein, which consists of 1582 amino acids. Primers were designed using ExonPrimer (www.ihg2.helmholtz-muenchen.de/ihg/ExonPrimer.html) and checked to exclude SNPs within the primer hybridization sites (www.ngrl.man.ac.uk/SNPCheck/Manchester).

The $A B C C 8$ gene was amplified by PCR using M13-tailed primers, and products were treated with Exonuclease I (New England Biolabs, Hitchin, United Kingdom) and Shrimp Alkaline Phosphatase (Promega, Southampton, United Kingdom) before sequencing in a single direction with an M13 sequencing primer. Two microliters of each PCR product was treated with 20 units of Exonuclease I and 1 unit of Shrimp Alkaline Phosphatase in a $3 \mu \mathrm{L}$ reaction volume. The sequencing reactions included $3 \mu \mathrm{L}$ of the PCR product mix, $0.37 \mu \mathrm{L}$ BigDye Terminator v3.1, $1.83 \mu \mathrm{L}$ BigDye dilution buffer (Applied Biosystems, Warrington, United Kingdom), $2 \mu \mathrm{L}$ M13 sequencing primer (MWG Biotech, Ebersberg, Germany), and $3.3 \mu \mathrm{L}$ water. Unincorporated nucleotides were removed using DTR Performa 96-well plates (VHBio, Gateshead, United Kingdom). Patient sequences were analyzed using Mutation Surveyor v3.10 by comparison with normal sequences (good quality sequences with no SNPs or mutations) and the reference GenBank sequence. Unidirectional analysis settings were utilized, and the ROI was also checked manually by examination of the electropherograms and difference traces. The ROI for each amplicon was defined as the $5^{\prime}$ conserved base of the branch site (nucleotide -30 to -50 of the preceding intron depending upon the location of the branch site, or -10 for exon 1 ) to +10 of the following intron; a total of 6473 nucleotides composed the ROI of the gene. 
PHRED scores were extracted from the ab1 sequence files by Certus Technology Associates Limited (Exeter, United Kingdom), and the mean PHRED scores for the ROI calculated. Further quality data for the ROI (quality score and average PHRED-like score) and the number of bases with a PHRED-like score $<20$ were obtained from the Mutation Surveyor HGVS table. The PHRED-like scores are calculated using a modified algorithm where one of the standard PHRED parameters, peak resolution, is not included.

\section{Results}

\section{Unidirectional sequence analysis for heterozygous base substitutions}

A total of 701 different heterozygous base substitutions in 29 genes were identified in patients referred for genetic testing to three clinical diagnostic laboratories. They included 495 pathogenic base substitutions, 12 unclassified variants, and 194 SNPs. All substitutions were detected by the Mutation Surveyor software. Therefore, the sensitivity of heterozygote detection was $\geq 99.57 \%$ (with $95 \%$ confidence). In addition, 130 heterozygous insertion/deletion mutations were detected by the software, and none were missed.

\section{Bidirectional sequence analysis for heterozygous base substitutions}

Our survey found no examples of base substitutions visible in only one direction. Four examples of heterozygous SNPs not detected by the Mutation Surveyor software in a single direction were reported by two laboratories out of a total of $\sim 11,000$ amplicons sequenced (0.04\%). In each case the base substitution was clearly visible (see Fig. 1A-D) although the INI1 and RYR2 SNP peaks were offset rather than superimposed (Fig. 1C-D). Analysis using the 1D settings enabled detection of the BRCA1 and BRCA2 SNPs but not the INI1 or RYR2 SNPs. The PHRED-like score for the INI1 and RYR2 heterozygous bases were 15 and 9, respectively (Fig. 1), lower than the threshold value of 20 utilized in the ABCC 8 data set to highlight lower quality bases.

\section{Analysis of unidirectional $A B C C 8$ sequence data}

A total of 13 pathogenic mutations (2 frameshifts and 11 base substitutions), 167 heterozygous SNPs, and 65 homozygous SNPs were identified by the Mutation Surveyor software in the 50 patient samples. No further variants were detected through visual inspection of the data.

The HGVS table highlighted that the ROI was not covered for the two amplicons containing the frameshift mutations or for most $(45 / 50)$ of the exon 16 sequences. Closer inspection revealed that the acceptable quality data (marked by a blue line on the GAD output) did not include the most $5^{\prime}$ nucleotide of the exon 16 ROI, and the forward primer has subsequently been redesigned.

Three quality parameters were examined for the ROI; Mutation Surveyor quality score, PHRED-like scores (derived from Mutation Surveyor), and PHRED scores. The Mutation Surveyor quality score is a measure of the signal-to-noise ratio where scores of 25 and 40 for the ROI represent $2 \%$ and $1.25 \%$ noise, respectively (Mutation Surveyor v3.10). The maximum quality score for the ROI is 50 , which is equivalent to $\leq 1 \%$ noise. The PHRED score is a measure of the accuracy of a base call where scores of 40 and 50 denote $99.99 \%$ and $99.999 \%$
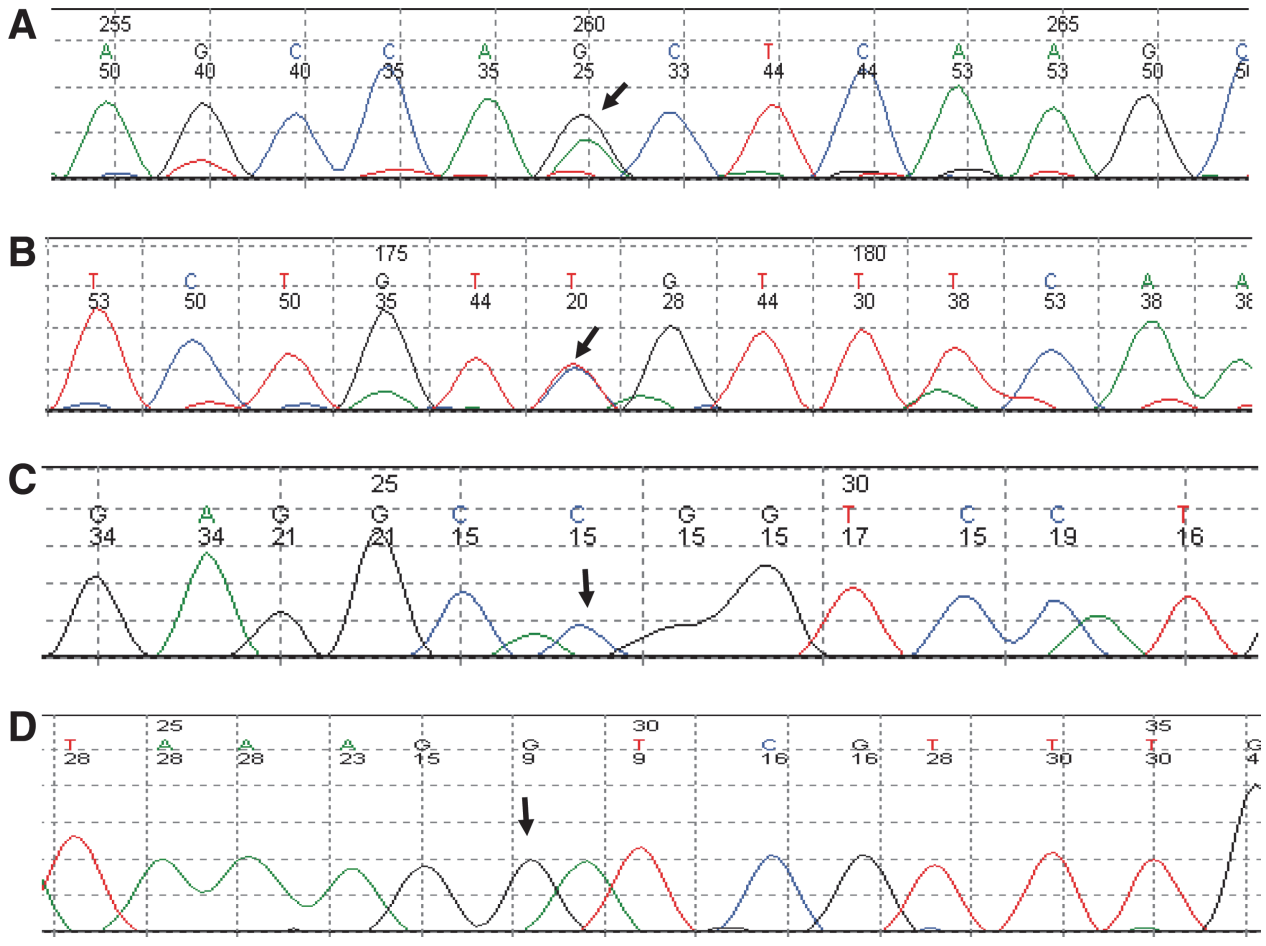

FIG. 1. Examples of heterozygous base substitutions not detected in one direction during bidirectional sequence analysis. Arrows show heterozygous bases, and PHRED-like scores are above the individual peaks. The heterozygous SNPs are (A) BRCA1 c.3119G >A; p.Ser1040Asn, (B) BRCA2 c.3807T>C; p.Val1283Val, (C) INI1 c.629-57C >A, and (D) RYR2 c.6556-41G>A. 


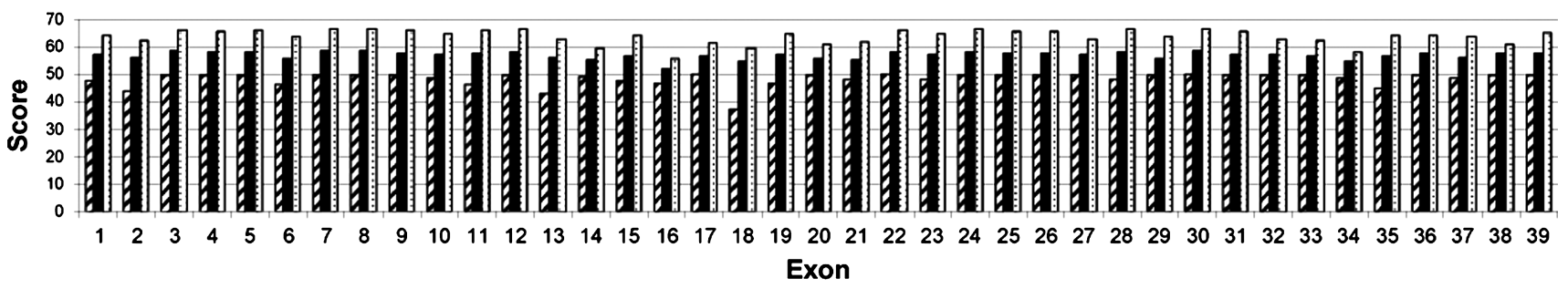

FIG. 2. Mean quality data for $A B C C 8$ exons 1-39 ROI ( $n=50$ patients per exon). Quality scores (diagonal striped bars), mean PHRED-like scores (solid bars), and mean PHRED scores (dotted bars) are shown.

accuracy. The Mutation Surveyor software calculates a PHRED-like score using a modified algorithm where one of the standard PHRED parameters, peak resolution, is not included.

Figure 2 illustrates the mean quality data ( $n=50$ patients) for each exon of the $A B C C 8$ data set. PHRED and PHRED-like scores were correlated $(r=0.9)$, and the PHRED-like scores were lower than the PHRED scores (overall mean 57.1 vs. 64). The mean PHRED-like score ranged from 52 (exon 16) to 58.8 (exon 8). A wider distribution of mean quality scores was observed, ranging from 37.3 (exon 18) to 50 (exon 22) with an overall mean of 48.3 .
The lowest individual amplicon quality score for the ROI was 11 , eight amplicons $(0.4 \%)$ had an ROI score $<30$, and 1007 sequences (52\%) achieved the maximum score of 50 . Three of the eight sequences with an ROI quality score $<30$ showed "n-1" peaks (Fig. 3D), and some were accepted because a pathogenic mutation (or mutations) confirming the diagnosis had been detected in another amplicon(s). Individual ROI PHRED-like scores ranged from 37 to 59 with $1900 / 1950(97.4 \%) \geq 50$. The number of amplicons with an ROI quality score $\geq 30$ and an average PHRED-like score $\geq 50$ was $1895(97.2 \%)$. The total number of nucleotides analyzed was 323,650 , and the percentage of bases with a PHRED score

A QS 50, mean PHRED-like 59, mean PHRED 67

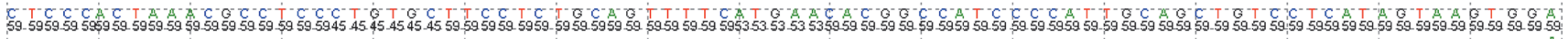

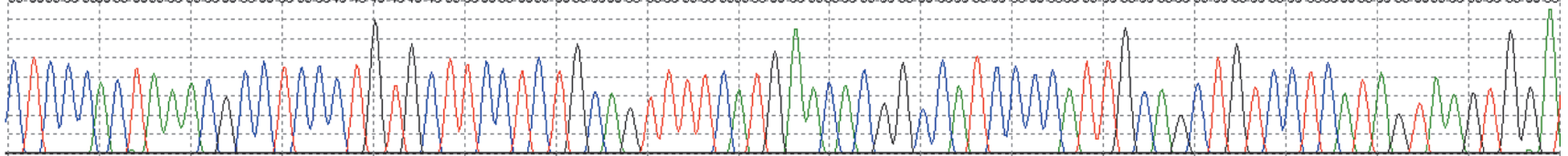

B QS 48, mean PHRED-like 57, mean PHRED 66

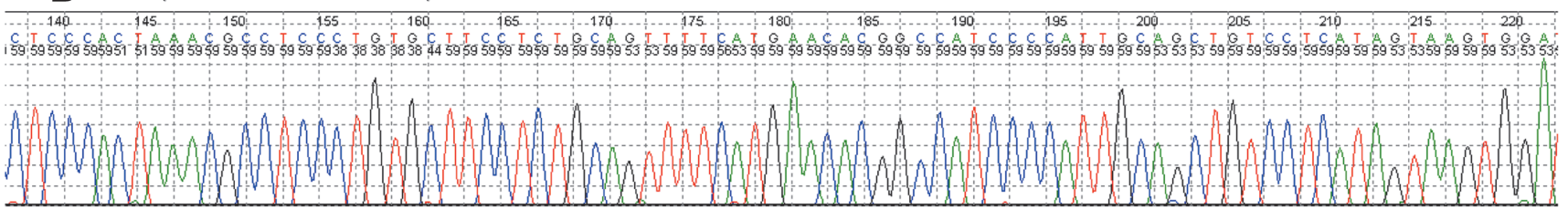
C QS 41, mean PHRED-like 57, mean PHRED 61

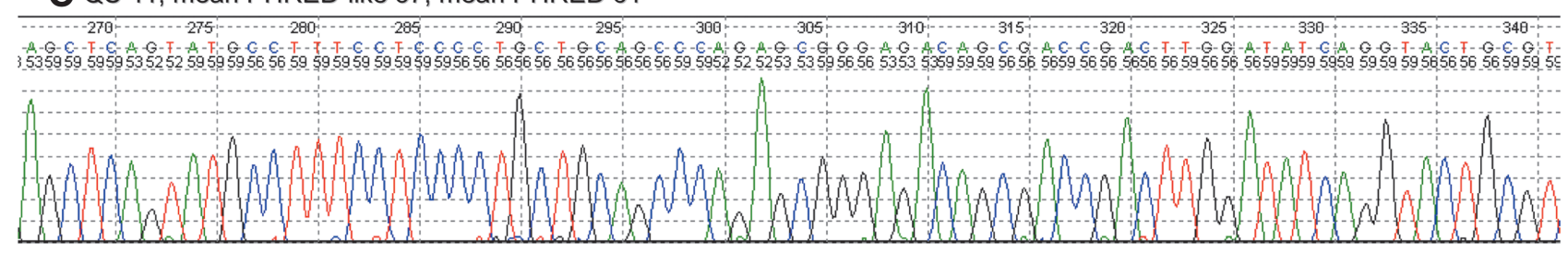

D QS 29, mean PHRED-like 54, mean PHRED 59

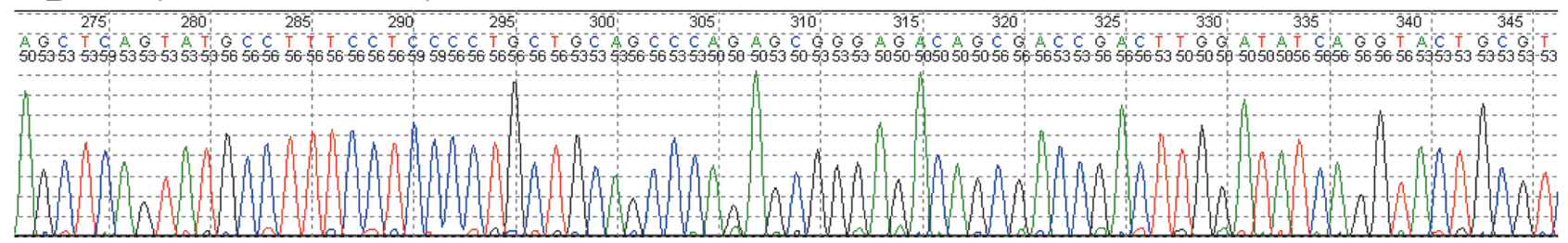

FIG. 3. Examples of sequence quality for $A B C C 8$ exons 11 and 18. Electropherograms for exon 11 (A, B) and exon 18 (C, D) ROI showing the ROI quality scores and individual base PHRED-like scores. The sequence shown in (B) is of similar quality to the data set (1950 amplicons) average of 48.3 (quality score), 57.1 (mean PHRED-like score), and 64 (mean PHRED score). The noise evident in sequence $(\mathbf{D})$ is consistent with low-level " $n-1$ " primer due either to unremoved by-product during synthesis or to primer degradation. 
exceeding 40,50 , and 55 was $98.1 \%, 88.1 \%$, and $86.1 \%$, respectively. Examples to illustrate the variability of quality and mean PHRED/PHRED-like scores are illustrated in Figure 3.

We selected a PHRED-like score of 20 as a threshold within the HGVS table options to highlight lower quality bases. This threshold was chosen (a) because a PHRED score of 20 is equivalent to a base call accuracy of $99 \%$ and (b) from previous analysis of sequences containing "dye blobs" or spiking caused by air bubbles where most affected bases had a score $\leq 15$ (data not shown). Of the 1950 sequences, 141 (7.2\%) included bases with a PHRED-like score $<20$. The number of individual bases not meeting this threshold was 419 (out of a total of 323,$650 ; 0.13 \%$ ), but 276 of these were bases adjacent to a heterozygous base substitution or within a frameshifted sequence.

\section{Discussion}

A number of sequence analysis software programs are available for assistance with mutation detection, but data regarding their sensitivity in the clinical diagnostic setting are scarce. We assessed the sensitivity of the Mutation Surveyor software package for the detection of heterozygous base substitutions in unidirectional sequence data. This collaborative study by three UK laboratories identified 701 different heterozygous base substitutions in 29 genes. Visual inspection of electropherograms failed to identify any variants not reported by the software, demonstrating that the sensitivity for heterozygote detection is at least $99.57 \%$ (with $95 \%$ confidence). This figure is higher than the manufacturer's quoted sensitivity of $95 \%$, and the false-negative rate of $0 \%$ is lower than that seen in the only previous evaluation of clinical diagnostic data (Patel and Wallace, 2005).

There is no known biological reason for a heterozygous base substitution being visible in only one direction, and bidirectional analysis is not always possible due to the presence of intronic insertion/deletion polymorphisms that generate frameshifted sequences in heterozygotes. Our survey of UK laboratories employing bidirectional sequence analysis yielded four examples where a heterozygous base was only detected in one direction. Two could be identified using the unidirectional analysis settings, which give greater sensitivity (but an increased false-positive rate). The remaining two examples occurred within 30 bases of the $5^{\prime}$ end of the sequence read, and there appears to be a mobility shift involving the variant base as evidenced by the overlapping peaks at nucleotides 32 and 33 in Figure 1C and the gap between nucleotides 33 and 34 in Figure 1D. However, these heterozygous bases would be highlighted for visual inspection if a threshold PHRED-like score of 20 was used to identify lower quality bases. Based on these data set it seems that there is no need for bidirectional sequencing if good quality sequence data are obtained for the ROI in one direction.

Analysis of the 1950 ABCC 8 sequences from a consecutive series of 50 patients demonstrated the high quality of sequence data that can be generated by capillary sequencing. In this data set $98.1 \%$ of the 323,650 bases examined had a PHRED score $>40$ compared to $64 \%$ of base calls from the sequences submitted to the 2003 European Quality Assurance scheme (Patton et al., 2006). In the report by Patton et al., the participating laboratories used a range of different sequencers and chemistries with the highest scoring laboratory achieving a PHRED score $>40$ for $84 \%$ of the bases examined. The improved quality demonstrated in the present study is partly attributable to the fact that our analysis was restricted to the ROI, and due to PCR primer design, poor quality bases at the start or end of the sequence read are automatically excluded. Optimization of both the primer sets and the sequencing protocol for maximal quality as defined by the signal:noise ratio also contribute to improved quality.

A recent addition to the Mutation Surveyor software is a feature that allows any bases within the ROI that fall below a user-defined threshold PHRED-like score to be listed in the HGVS table. We found 419 bases (out of 323,$650 ; 0.13 \%$ ) that had PHRED-like scores $<20$. Nearly two thirds of these bases were either within a frameshifted sequence or adjacent to a heterozygous base substitution and would have already been examined during inspection of variants highlighted within the Mutation Report.

Some visual inspection will always be required in a clinical diagnostic setting to confirm the presence of a mutation and its correct description, and to check individual bases with low quality scores. Sequences that fail to meet minimum ROI quality scores must be examined to determine whether repeat testing is necessary or if visual inspection can suffice. Further work is required to define the thresholds for visual inspection of individual bases or sequences, and to decide for the latter whether a score based on signal:noise ratio or average PHRED (or PHRED-like) score is most informative.

It should be noted that low-level somatic mosaic mutations are unlikely to be detected by semiautomated analysis of unidirectional sequence data. For disorders such as Neurofibromatosis type 2 and Tuberous Sclerosis where somatic mosaicism in clinically affected cases is not an infrequent finding (Verhoef et al., 1999; Moyhuddin et al., 2003), bidirectional analysis using the settings for mosaic mutation detection may be most appropriate. However, somatic mosaicism (at a level $\geq 5 \%$ ) in an unaffected parent is likely to be recognized during routine comparison of electropherograms with that from the affected child. Laboratories should also be aware that a missense mutation downstream of a frameshift is likely to remain undetected during unidirectional sequence analysis. Hence, if a single heterozygous frameshift mutation is detected in a patient affected with a recessively inherited disorder, we recommend that either the sequence is manually inspected for the presence of a missense mutation in the frameshifted region, the amplicon is sequenced in the reverse direction, or parental samples are analyzed.

We estimate that a semiautomated sequence analysis strategy where visual inspection is confined to the checking of mutations, polymorphisms and low quality bases or sequences may reduce the time required for visual inspection by up to $50 \%$. Prescreening methods for mutation detection such as denaturing high performance liquid chromatography, conformation-sensitive capillary electrophoresis, and highresolution melt-curve analysis are sometimes preferred to sequencing because of the cheaper consumable costs. However, the most expensive component of a test is the labor cost, and automated analysis for these methods is not currently validated for diagnostic use. Semiautomated unidirectional sequence analysis may therefore be a cost-effective option with both consumable and labor costs reduced in comparison to manual examination of bidirectional sequencing. Sequencing is particularly suitable for the analysis of polymorphic genes 
(such as $A B C C 8$ ), and once a laboratory has optimized their sequencing protocol to achieve high quality data, developing sequence-based mutation scanning for additional genes is relatively fast. For a lab analyzing a large number of different genes, a sequencing pipeline approach may prove more economic.

In summary, we propose that bidirectional sequence analysis is not necessary for heterozygote detection if high quality sequence data are available for the defined ROI (usually the coding region and conserved splice sites). Semiautomated analysis of good quality unidirectional sequence data has high sensitivity $(>99 \%)$ and is suitable for mutation screening in clinical diagnostic laboratories.

\section{Acknowledgments}

We thank Piers Fulton, Michael Day, Dr. Ann-Marie Patch, Kevin Colclough, and Dr. Mike Weedon for their contributions to this study. The technical expertise of Jonathan Liu and colleagues at SoftGenetics and their willingness to adapt the Mutation Surveyor package to meet the needs of the UK NHS molecular genetics laboratories is appreciated. Financial support for this study was provided by the National Genetics Reference Laboratory (Manchester), the Wellcome Trust (081188/A/06/Z), and the Research \& Development Directorate at the Royal Devon \& Exeter NHS Foundation Trust.

\section{Disclosure Statement}

No competing financial interests exist.

\section{References}

Ellard S, Flanagan SE, Girard CA, et al. (2007) Permanent neonatal diabetes caused by dominant, recessive, or compound heterozygous SUR1 mutations with opposite functional effects. Am J Hum Genet 81:375-382.

Flanagan SE, Patch AM, Mackay DJ, et al. (2007) Mutations in ATP-sensitive $\mathrm{K}+$ channel genes cause transient neonatal diabetes and permanent diabetes in childhood or adulthood. Diabetes 56:1930-1937.

McKusick VA (2007) Mendelian inheritance in man and its online version, OMIM. Am J Hum Genet 80:588-604.

Moyhuddin A, Baser ME, Watson C, et al. (2003) Somatic mosaicism in neurofibromatosis 2: prevalence and risk of disease transmission to offspring. J Med Genet 40:459-463.

Patel Y, Wallace A (2005) Automated mutation detection using SoftGenetics Mutation Surveyor v2.51. http://www.ngrl.org .uk/Manchester/Technologypubs.htm.

Patton SJ, Wallace AJ, Elles R (2006) Benchmark for evaluating the quality of DNA sequencing: proposal from an international external quality assessment scheme. Clin Chem 52:728736.

Steensma DP (2006) JAK2 V617F in myeloid disorders: molecular diagnostic techniques and their clinical utility: a paper from the 2005 William Beaumont Hospital Symposium on Molecular Pathology. J Mol Diagn 8:397-411.

Thomas P, Ye Y, Lightner E (1996) Mutation of the pancreatic islet inward rectifier Kir6.2 also leads to familial persistent hyperinsulinemic hypoglycemia of infancy. Hum Mol Genet 5:1809-1812.

Thomas PM, Cote GJ, Wohllk N, et al. (1995) Mutations in the sulfonylurea receptor gene in familial persistent hyperinsulinemic hypoglycemia of infancy. Science 268:426-429.

Verhoef S, Bakker L, Tempelaars AM, et al. (1999) High rate of mosaicism in tuberous sclerosis complex. Am J Hum Genet 64:1632-1637.

Address reprint requests to: Sian Ellard, Ph.D. Department of Molecular Genetics Royal Devon E Exeter NHS Foundation Trust Barrack Road Exeter EX2 5DW United Kingdom

E-mail: sian.ellard@rdeft.nhs.uk 